\title{
Time resolved tunable diode laser absoption spectroscopy of dual High Power Impulse Magnetron Sputtering discharges
}

\author{
Hoang Tung Do \\ Laboratory for Plasma Technology, Center for Engineering Physics, Institute of Physics, \\ Vietnam Academy of Science and Technology, No. 10 Dao Tan, Ba Dinh, Ha Noi, Vietnam \\ dhtung@iop.vast.ac.vn \\ Vitezslav Stranak \\ Interface Physics Laboratory, Institute for Physics, University of Greifswald \\ Felix-Hausdorff Strasse 6, 17489 Greifswald, Germany \\ stranv00@centrum.cz. \\ Rainer Hippler \\ Interface Physics Laboratory, Institute for Physics, University of Greifswald \\ Felix-Hausdorff Strasse 6, 17489 Greifswald, Germany \\ hippler@physik.uni-greifswald.de \\ Published 13 August 2014
}

\begin{abstract}
Time-resolved measurements have been performed during dual High Power Impulse Magnetron Sputtering (dual-HiPIMS) with two cathodes in a closed magnetic field configuration. The dualHiPIMS system, operated at a repetition frequency $\mathrm{f}=100 \mathrm{~Hz}$ and duty cycle of $1 \%$, was equipped with two different metallic targets $(\mathrm{Ti}, \mathrm{Cu}$ ). The effect of a delay between subsequent pulses on argon excited atom density and temperature was investigated by means of tunable diode laser absorption spectroscopy. It is shown that the peak densities of pulses vary strongly with the delay. We observed an enhancement of metastable density due to pre-ionization effect but more effective than that is the contribution of metal atoms which have smaller ionization energy compare to that of buffer gas atom. Associate with the enhancement of density, the temporal variation of metastable atom temperature in the $\mathrm{Cu}$ pulse also transforms from those of low current pulse into the high current one.
\end{abstract}

Keywords: HiPIMS; TR-TDLAS; metastable.

\section{Introduction}

Magnetron sputtering (MS) deposition is a plasma-based coating technique well known ${ }^{1,2}$ since 1980. Inert gas ions (usually $\mathrm{Ar}^{+}$) are accelerated to bombard cathode/target made

This is an Open Access article published by World Scientific Publishing Company. It is distributed under the terms of the Creative Commons Attribution 3.0 (CC-BY) License. Further distribution of this work is permitted, provided the original work is properly cited.

*Institute of Physics and Biophysics, University of South Bohemia, Branisovska 31, 37005 Ceske Budejovice, Czech Republic. 
from the material to be deposited. Sputtered target atoms condensate onto a substrate and form a film. Growth and formation of the film are strongly influenced by ion and energy fluxes to the substrate. ${ }^{3,4}$ High ionization degree of sputtered particles and high ion fluxes can be achieved in so-called high power impulse MS (HiPIMS) discharges ${ }^{5}$ working at low frequency $(f \sim 100 \mathrm{~Hz})$ and short pulse width $\left(T_{\mathrm{a}} \approx 10-100 \mu \mathrm{s}\right)$. HiPIMS discharges have high power pulse density (approximately kilowatt per centimeter square) and high concentration of charged particles ${ }^{6}$ (typically $\geq 10^{18} \mathrm{~m}^{-3}$ ).

However, deposition rate in HiPIMS is rather low due to its high ionization degree of sputtered particles. ${ }^{7}$ This is one of the limiting parameters for wide application of HiPIMS in the coating industry. Many attempts have been made to overcome this limitation. One of those is the HiPIMS discharges working with higher repetition frequency $(f \geq 1 \mathrm{kHz})$ and longer pulse widths $\left(T_{\mathrm{a}} \approx 100-400 \mu \mathrm{s}\right){ }^{8},{ }^{9}$ In such configuration the HiPIMS advantages are preserved and in addition the higher deposition rate $(\sim 2 \mu \mathrm{m} / \mathrm{min})$, which cannot be achieved by a conventional dc MS due to a target overheating, have been observed. Other efficient sputtering systems employing two magnetrons equipped with the same target material and synchronously applying the same cathode voltage have been developed in the last years, too. The dual-HiPIMS with openmagnetic field, employing two identical target materials ${ }^{10}$ and the so-called dual-MS with closed magnetic field (two magnetrons with opposite polarity of magnetic field ). ${ }^{11,12}$ Recently, a more flexible system is proposed by Stranak et al. and co-workers ${ }^{13}$ using a dual magnetron systems with closed magnetic field working at repetition frequency $f=$ $100 \mathrm{~Hz}$ and pulse width $T_{\mathrm{a}}=100 \mu \mathrm{s}$. In this system, the sputtering sources were equipped with different target materials $(\mathrm{Cu}, \mathrm{Ti})$ and driven at different cathode voltages to optimize the thin film stoichiometry. The delay between subsequent pulses, i.e. the delay between a Ti pulses followed by a $\mathrm{Cu}$ pulse, can also be varied to control the parameters responsible for thin film growth.

The influence of time delay on plasma behavior of dual HiPIMS system was first studied in. ${ }^{13}$ It was noted that the pre-ionization effect promoting the ignition of the subsequent pulse which result in a higher plasma density and smaller electron temperature. The role of metastable atoms in noble gas discharges have fundamental importance from the point of view of discharge sustaining, kinetics of excited species and influence on the electron distribution function. In gas mixtures, the collisions with metastables cause large range of reactions, including Penning ionization and chemical transformations. Metastable are abundantly available in post-discharge ${ }^{14}$ and may contribute also to the fast ignition of the second pulse.

Tunable diode laser absorption spectroscopy (TDLAS) show capability to diagnose time resolvedly both buffer gas metastable atom density and temperature ${ }^{14,15}$ as well as sputtered atom ${ }^{14}$ in HiPIMS discharges. Therefore, it is worthy using time resolved TDLAS in order to get insight into the influence of pulse delay on plasma properties of dual HiPIMS. 


\section{Experiment and Diagnostic Method}

\subsection{Experimental setup}

The experimental set-up has been described in detail elsewhere. ${ }^{16}$ It consists of a vacuum chamber pumped by a turbomolecular pump to a base pressure of better than $10^{-5} \mathrm{~Pa}$. A dual-HiPIMS system with closed magnetic field has been developed for deposition of Ti$\mathrm{Cu}$ films. Two commercial planar magnetrons (Gencoa, Liverpool, UK), with opposite magnetic field configurations, are attached to the upper flange of the vacuum chamber and located $11 \mathrm{~cm}$ below the flange. The magnetrons are equipped with $\mathrm{Ti}$ and $\mathrm{Cu}$ targets $50 \mathrm{~mm}$ in diameter. The working pressure is adjusted by a throttle valve installed between the vacuum chamber and the pump. All experiments were carried out in an argon atmosphere at a pressure $p=3 \mathrm{~Pa}$; the argon flow rate of $20 \mathrm{sccm}$ was controlled by a mass flow controller.

The high power electrical circuit of the dual sputtering system is based on a parallel combination of two identical pulsed power switches as employed in our previous works. ${ }^{13,17,18}$ Each of the 2 electric loops employs a power switch in combination with a commercial direct current power supply (AE Pinnacle 3000) with a range down to -1000 $\mathrm{V}$ designed for continuous operation and used to charge a large capacitor during the idle part of the pulses. A ballast resistor $(R=2.1 \Omega)$ serving without magnetron discharge as a dummy load was inserted in series with the magnetron cathode path. A home-built signal wave-form generator, employed to set repetition frequency $f$, pulse width $T_{\mathrm{a}}$, and delay between subsequent pulses, was employed for activation of the power switches and to trigger the time-resolved measurements. Measurements with different delays between subsequent pulses were carried out. Mean discharge currents $I_{\mathrm{Ti}}=400 \mathrm{~mA}$ and $I_{\mathrm{Cu}}=$ $100 \mathrm{~mA}$ were kept constant during the experiments.

\subsection{Time resolved TDLAS}

Time-resolved light absorption spectroscopy was performed with the help of a tunable diode laser. The single mode diode laser (Toptica DL100) is equipped with an external cavity. The spectral band width is much smaller than the width the absorption profile in a low pressure glow discharge. The laser light beam is split into two parts with a beam splitter. The first beam is guided to a confocal Fabry-Perot interferometer with $1 \mathrm{GHz}$ free spectral range and detected with the first photo-diode to perform a calibration of the laser frequency scan. The second beam is launched into the discharge chamber where it passes parallel to the target and is detected on the opposite side of the chamber with the second photodiode. Measurements were carried out at a distance of $10 \mathrm{~cm}$ from the cathode where the substrate was used to be located. The diode laser was used for the argon $1 \mathrm{~s}_{5}-2 \mathrm{p}_{9}$ transition (in Paschen's notation) with a center wavelength of $811.53 \mathrm{~nm} .{ }^{19}$ Measurements were carried out at sufficiently low laser powers where saturation effects are negligible $(<5 \%)$. 

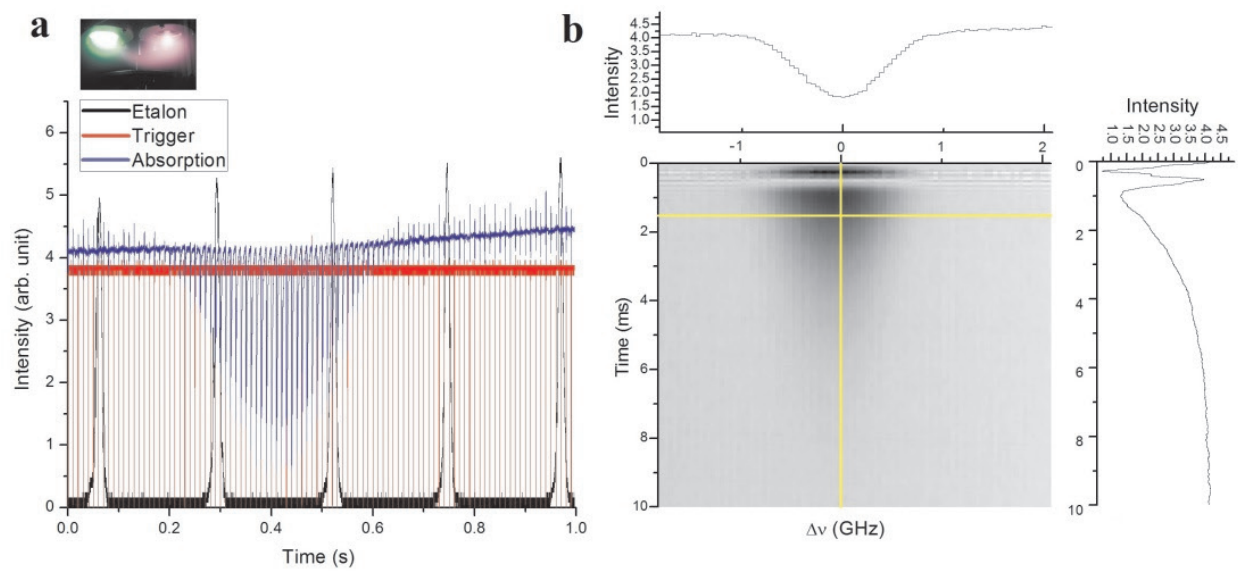

Fig. 1. Data acquisition (a) as measured, (b) reconstructed.

The laser diode scan frequency was fixed at $0.5 \mathrm{~Hz}$, so that during one half period of the wavelength scan 100 plasma pulses may occur. Three channels were recorded into the oscilloscope: the etalon signal (black curve, figure 1a), the absorption signal (blue curve, Fig. 1a), and the trigger signal of Ti magnetron (red curve, Fig. 1a). The absorption signal was first subtracted by the offset, then rescaled from time scale into frequency scale using etalon signal as reference. The rescaled absorption signal was subsequently be reconstructed with regard to the triggering of magnetron to form time resolved absorption profiles of every time point in the discharge period as shown in figure 1b. From these time resolved absorption profile one can easily calculate the time resolved temperature and density of argon metastable using the analyzing sequence described in. ${ }^{20}$

\section{Results and Discussions}

\subsection{Time resolve metastable density and temperature in dual-HiPIMS}

Typical time-resolved argon metastable density and temperature during dual-HiPIMS are displayed in Fig. 2. Each pulse ( $\mathrm{Ti}$, and $\mathrm{Cu}$ ) behaves almost similar to an ordinary HiPIMS discharge. The argon metastable density features a double peak structure for each pulse where the first maximum occurring during the pulse is followed by a second maximum in the afterglow. Both regimes are well separated from each other. The first maximum during the pulse is caused by electron impact excitation of argon atoms. The rapidly decreasing of metastable density in the afterglow after the plasma is switched off is largely caused by de-excitation due to electron impact. The following second maximum in the afterglow can be explained by electron-ion recombination giving rise to the formation of excited argon atoms (Ar*) which eventually decay to the metastable state. $^{14,21,22}$ 


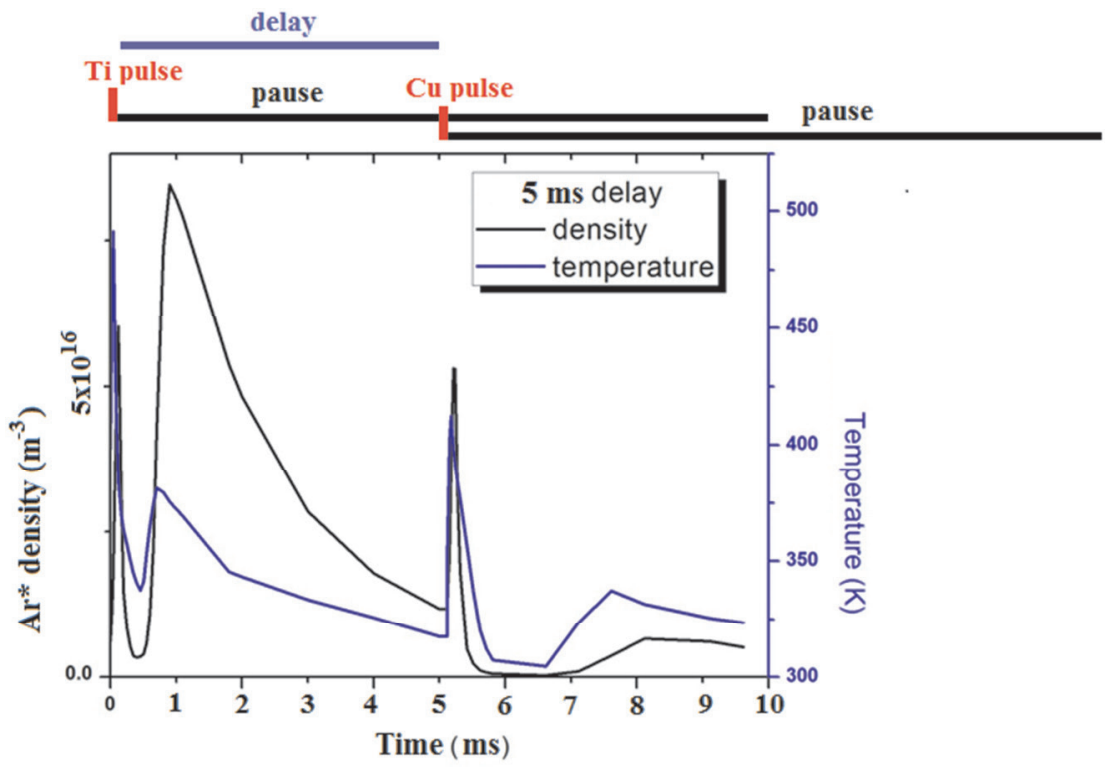

Fig. 2. Time resolved density and temperature of argon metastable in a dual-HiPIMS discharge with $\mathrm{Ti}$ and $\mathrm{Cu}$ as target materials. The time delay between the two pulses is $5 \mathrm{~ms}$.

The time dependence of temperature is quite similar to the temporal evolution of the metastable density. The temperature displays a pronounced increase of about $200 \mathrm{~K}$ during the plasma on phase followed by a fast decrease in the afterglow and a subsequent moderate increase. The first rise correlates with the increase in the Ar metastable density; it is presumably caused by the heating of the plasma due to ions, fast neutralized argon ions reflected by the cathode, and the energy released by sputtered atoms. The second temperature rise during the afterglow reflects the ion recombination effect. The exact heating mechanism is not clear, however. It appears that ions are not fully thermalized and, as a consequence, the metastable atoms forming from these ions by recombination thus attain a higher temperature compared with the surrounding gas atoms.

\subsection{Influence of delay on metastable density}

Figure 3 displays peak densities of the first density peaks of $\mathrm{Ti}$ and $\mathrm{Cu}$ pulses in different discharges with different delays. At a first glance, one can see a very strong variation of peak density of $\mathrm{Cu}$ pulse (almost 350\%) and a moderate variation of Ti pulse (about 148\%). The original HiPIMS peak density for Ti (black solid curve, Fig. 3) and $\mathrm{Cu}$ (red solid curve, Fig. 3) are also displayed in Fig. 3. There is clearly positive influence of the dual pulse system as the corresponding peak density of dual HiPIMS is always higher than that of the ordinary one. Pre-ionization effect manifests itself in an elevation of $\mathrm{Cu}$ peak density of about $30 \%$ for $15 \mu$ s delay. The peak density of $\mathrm{Cu}$ pulse first decreases with longer delay as the pre-ionization effect would become smaller. ${ }^{13}$ However, for delays longer than $4 \mathrm{~ms}$ the enhancement effect become more and more pronounce 
reaching the maximum at around $7 \mathrm{~ms}$. The behavior of peak density of Ti pulse, in general, is similar to that of $\mathrm{Cu}$ pulse, except two noticeable differences: the preionization effect is not well to be seen and the maximum increase appears far earlier at around $1 \mathrm{~ms}$ delay.

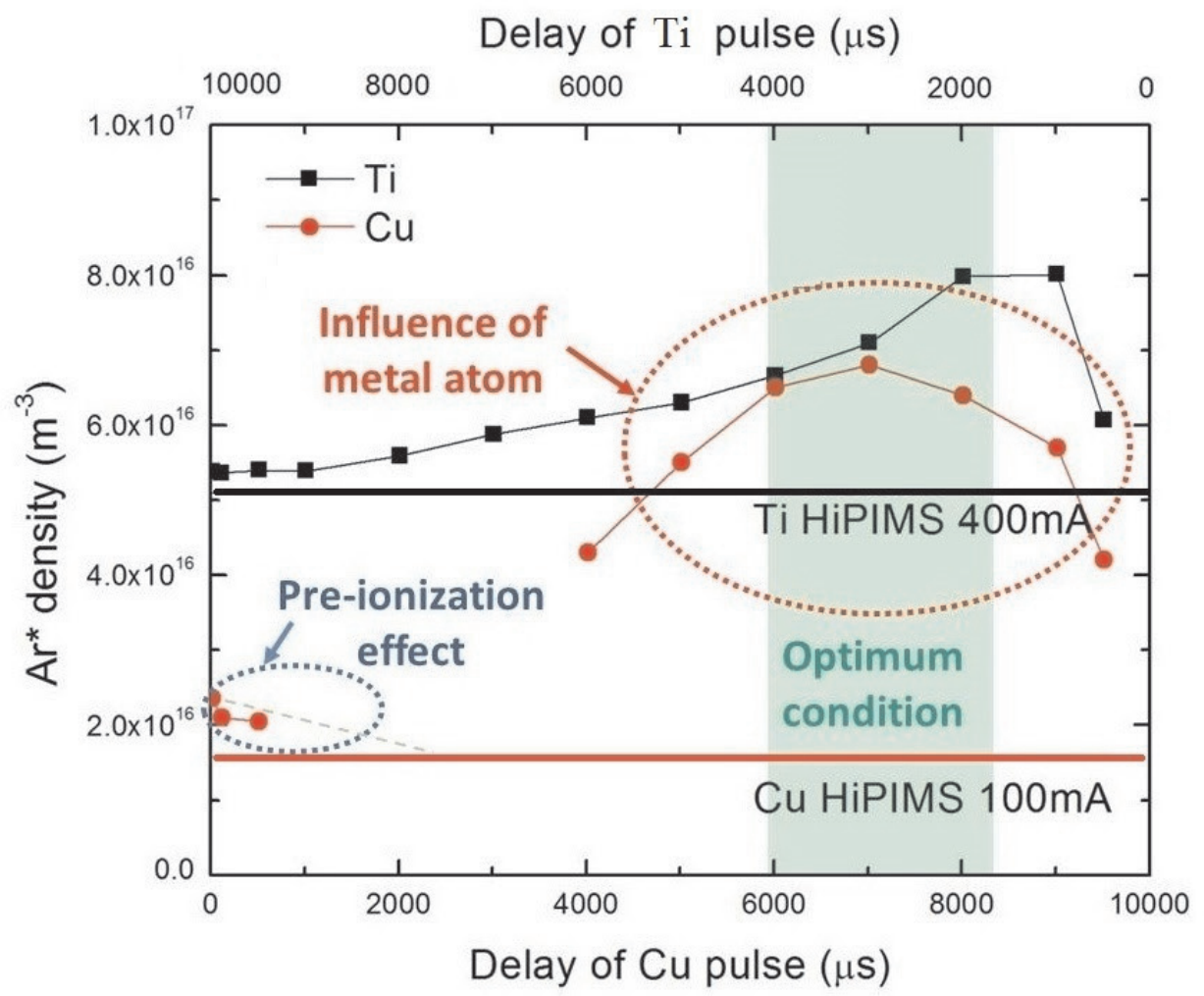

Fig. 3. Dependence of peak density of the first density peak of metastable density on the delay between subsequence pulses.

The strong enhancement of metastable density in the metastable density could be attributed to the presence of metal atom sputtered from previous pulse which promotes the ignition of the subsequence pulse due to their much lower ionization energy (in comparison to Ar). To support this argument, let us consider the metal atom density measured in the work, ${ }^{14}$ for a $100 \mathrm{~mA}$ averaged discharge current of HiPIMS with Ti as target material. When the discharge was switched off, the Ti atom density rapidly increases to a maximum at around $1 \mathrm{~ms}$ then slowly decreases. The $\mathrm{Cu}$ pulse in our dual HiPIMS discharge which has the same averaged discharge current would be expected to provide a similar behavior of $\mathrm{Cu}$ atom density. This behavior also resembles the behavior of peak density of Ti pulse as shown in figure 3 above. In line with this reasoning, the Ti pulse in our dual HiPIMS discharge has much higher discharge current and of course a higher degree of ionization. The maximum of Ti atom density after the Ti pulse was 
switched off would appear later and so the maximum of peak density of argon metastable of $\mathrm{Cu}$ pulse.

\subsection{Influence of delay on metastable atom temperature}

Figure 4 shows the temporal behavior of metastable atom temperature in the Ti pulse with two different delay times $1 \mathrm{~ms}$ and $7 \mathrm{~ms}$.

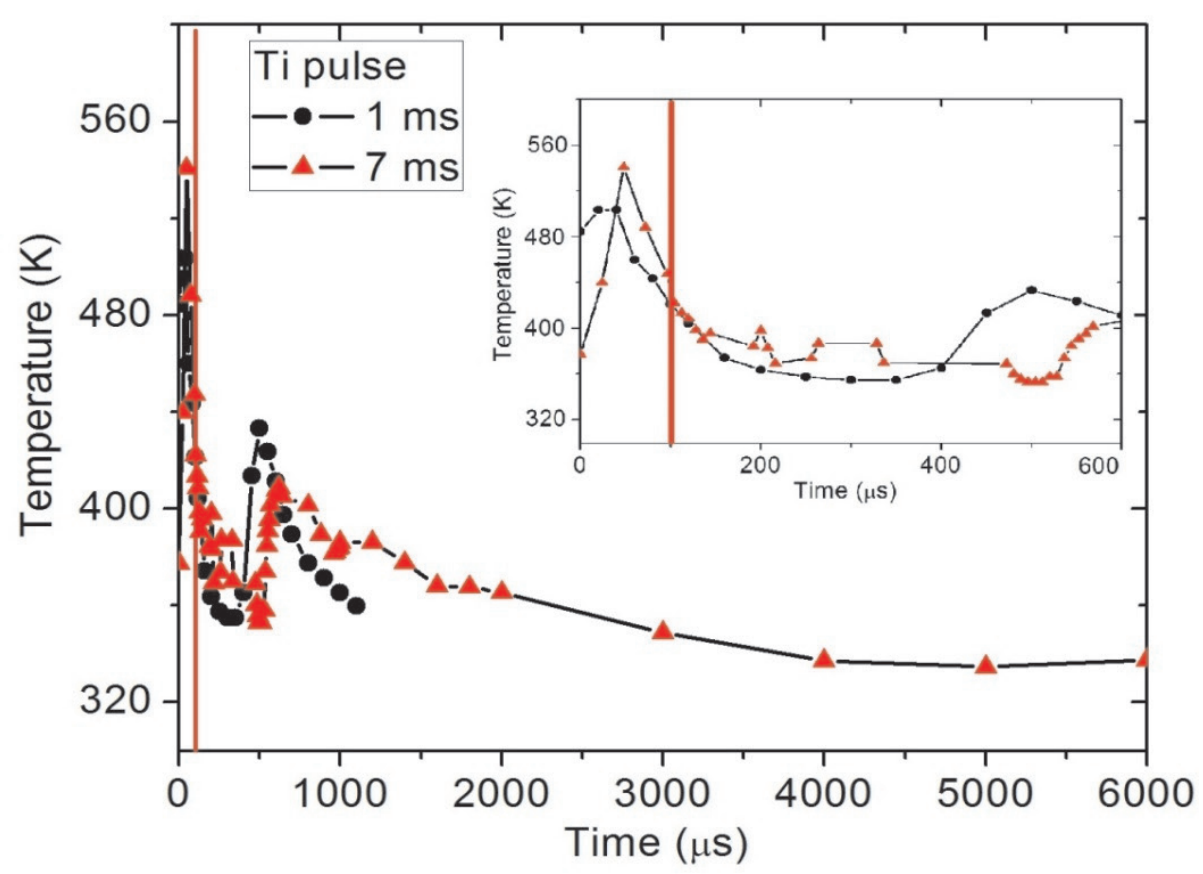

Fig. 4. Temporal behavior of metastable atom temperature of Ti pulse with two different delay times (delay of the $\mathrm{Cu}$ pulse to the Ti pulse). The red vertical line marks the end of the active Ti pulse.

It is noted that the metastable temperature similar to the behavior of the metastable density reaches its maximum of about $500 \mathrm{~K}$ already during the pulse. This reflects the transition from an argon-burning to a titanium-burning discharge with a lower electron temperature resulting in a ceasing metastable production. The decreasing temperature at the end of the plasma on phase and in the early afterglow follows the decreasing metastable density.

Figure 5 shows the temporal behavior of metastable atom temperature in the $\mathrm{Cu}$ pulse with different delay times. The peak temperature follows the behavior of peak density, for higher peak density the peak temperature also higher and appears earlier. The temperature peak of the $\mathrm{Cu}$ pulse with short delay appears after the end of the active pulse. However, with delay time longer than $4 \mathrm{~ms}$ it comes before and has the shape almost similar to that of $\mathrm{Ti}$ pulse which indeed has its plasma current four time higher. This supports the statement above that the presence of metal atoms promotes the ignition 
of the plasma which eventually develop to a stronger plasma which in turn gives higher atom temperature and density.

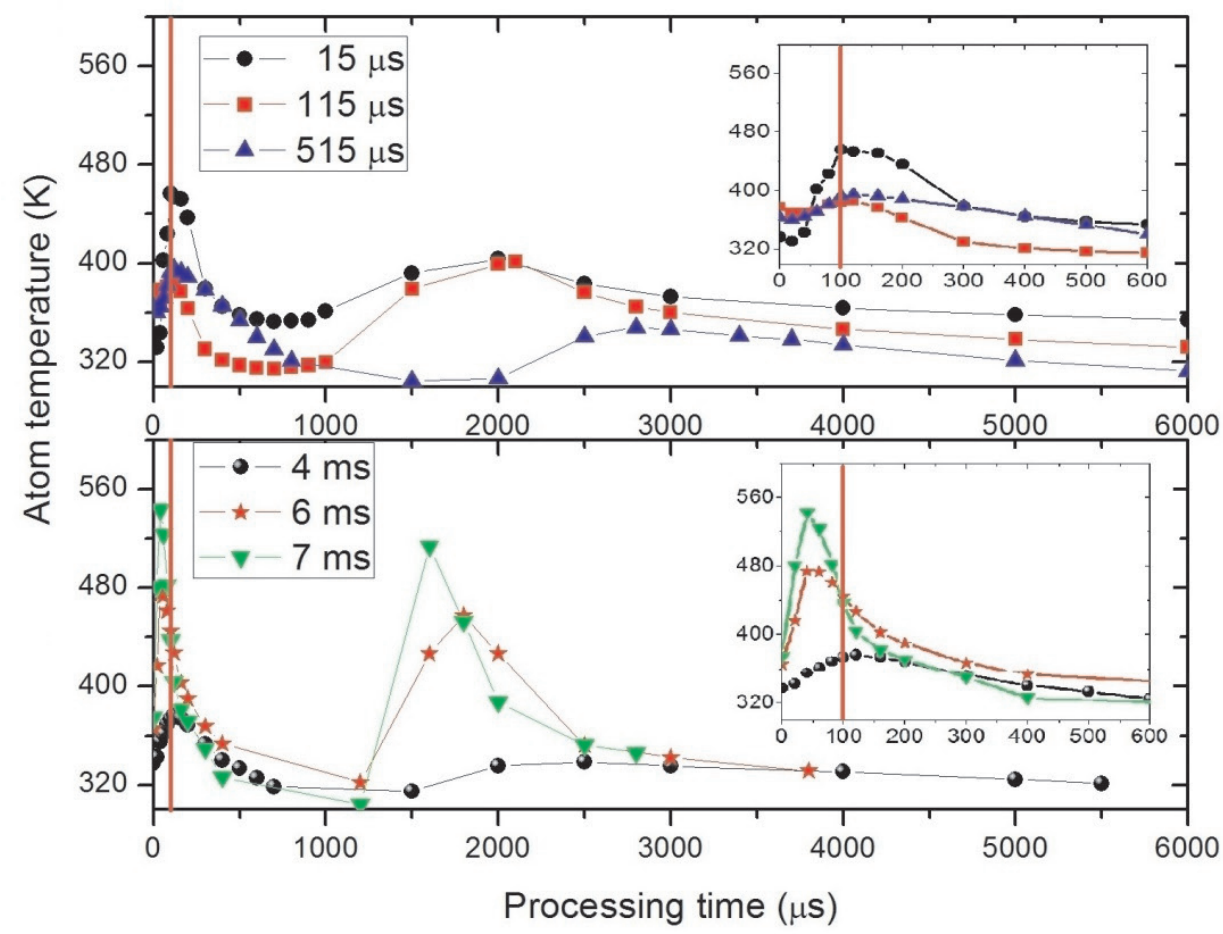

Fig. 5. Temporal behavior of metastable atom temperature of $\mathrm{Cu}$ pulse with different delay times (delay of the $\mathrm{Cu}$ pulse to the Ti pulse). The red vertical line marks the end of the active $\mathrm{Cu}$ pulse.

\section{Conclusions}

We performed a time-resolved tunable diode laser absorption spectroscopy to measurement argon metastable atom density and temperature in dual high power impulse magnetron sputtering discharges with two cathodes in a closed magnetic field configuration. Each pulse in a dual-HiPIMS feature almost similar behavior of density as well as temperature of metastable in a single HiPIMS. Mutual influence between pulses was demonstrated undoubtedly in dual-HiPIMS plasma. In out plasma condition, the most effective delay configuration is $7 \mathrm{~ms}$. The presence of sputtered metal atom in the gas phase promotes the ignition and behavior of HiPIMS pulses.

\section{Acknowledgments}

This work was supported by the Deutsche Forschungsgemeinschaft through SFB/TR 24, by the Czech Science Foundation through GACR P205/11/0386 and P108/12/2104 and by the National Foundation for Science and Technology Development (NAFOSTED), Vietnam, under project number 103.05-2012.20. 


\section{References}

1. R. K. Waits, J. Vac. Sci. Technol. 15, 179 (1978).

2. K. Ellmer, in Low Temperature Plasmas, edited by R. Hippler, H. Kersten, M. Schmidt, and K. H. Schoenbach (Wiley, New York, 2009), Vol. 2, p. 675.

3. I. Petrov, P.B. Barna, L. Hultman, J.E. Greene, J. Vac. Sci. Technol. 21, 117 (2003).

4. V. Stranak, M. Cada, M. Quaas, S. Block, R. Bogdanowicz, S. Kment, H. Wulff, Z. Hubicka, C.A. Helm, M. Tichy, R. Hippler, J. Phys. D: Appl. Phys. 42, 105204 (2009).

5. J. Bohlmark, M. Lattemann, J. T. Gudmundsson, A. P. Ehiasarian, Y. A. Gonzalvo, N. Brenning, and U. Helmersson, Thin Solid Films 515, 1522 (2006).

6. U. Helmersson, M. Lattemann, J. Bohlmark, A. P. Ehiasarian, and J. T. Gudmundsson, Thin Solid Films 513,1 (2006).

7. K. Sarakinos, J. Alami, and S. Konstantinidis, Surf. Coat. Technol. 204, 1661 (2010).

8. J. Vlček, A. D. Pajdarová, and J. Musil, Contrib. Plasma Phys. 44, 426 (2004).

9. A. Anders, J. Andersson, and A. Ehiasarian, J. Appl. Phys. 102, 113303 (2007).

10. A. Aijaz, D. Lundin, P. Larsson, and U. Helmersson, Surf. Coat. Technol. 204, 2165 (2010).

11. J. Musil, P. Baroch, J. Vlcek, K. H. Nam, and J. G. Han, Thin Solid Films 475, 208 (2005).

12. A. Bogaerts, E. Bultinck, I. Kolev, L. Schwaederlé, K. V. Aeken, G. Buyle, and D. Depla, J. Phys. D: Appl. Phys. 42, 194018 (2009).

13. V. Stranak, S. Drache, M. Cada, Z. Hubicka, M.Tichy, and R. Hippler, Contrib. Plasma Phys. 51, $237-245$ (2011).

14. V Sushkov, HT Do, M Cada, Z Hubicka and R Hippler, Plasma Sources Sci. Technol. 22, 015002 (2013).

15. C Vitelaru, D Lundin, G D Stancu, N Brenning, J Bretagne and T Minea, Plasma Sources Sci. Technol. 21, 025010 (2012).

16. V. Stranak, M. Cada, Z. Hubicka, M. Tichy, R. Hippler, J. Phys. D: Appl. Phys. 108, 043305 (2010).

17. V. Stranak, M. Quaas, H. Wulff, Z. Hubicka, S. Wrehde, M. Tichy, R. Hippler, J. Phys. D: Appl. Phys. 41, 055202 (2008).

18. S. Pfau, M. Tichy, Langmuir probe diagnostics; in: Low Temperature Plasma Physics, R. Hippler, H. Kersten, M. Schmidt, K.-H. Schoenbach, Eds., Wiley-VCH, Berlin, 175 (2008).

19. Y. Ralchenko, A. E. Kramida, J. Reader and NIST ASD Team 2011 NIST Atomic Spectra Database (ver. 4.1.0) (Gaithersburg, MD: NIST) http://physics.nist.gov/asd

20. H. T. Do, V. Sushkov and R. Hippler, New Journal of Physics 11, 03302 (2009).

21. A. Bogaerts, J. Anal. At. Spectrom. 22, 502 (2007).

22. N. Nafarizal, N. Takada and K. Sasaki, J. Phys. D: Appl. Phys. 41, 035206 (2008). 